Letters to the Editor

\title{
Feline Infectious Peritonitis and Feline Coronavirus Interest During the COVID-19 Pandemic: A Google Trends Analysis
}

\author{
${ }^{1}$ Mohammad Jokar, ${ }^{2}$ Vahid Rahmanian, ${ }^{2}$ Nader Sharifi, ${ }^{2}$ Narges Rahmanian and ${ }^{3}$ Hekmatollah Khoubfekr \\ ${ }^{I}$ DVM, Faculty of Veterinary Medicine, Karaj Branch, Islamic Azad University, Karaj, Iran \\ ${ }^{2}$ Zoonoses Research Center, Jahrom University of Medical Sciences, Jahrom, Iran \\ ${ }^{3}$ Iranshahr Health Services, Iranshahr University of Medical Sciences, Iranshahr, Iran
}

\author{
Article history \\ Received: 04-02-2021 \\ Revised: 10-05-2021 \\ Accepted: 18-05-2021 \\ Corresponding Author: \\ Vahid Rahmanian \\ Zoonoses Research Center, \\ Jahrom University of Medical \\ Sciences, Jahrom, Iran \\ Email: rahmanian.vahid@ut.ac.ir
}

\begin{abstract}
Coronaviruses infected different species of animals, such as feline, canine, swine and bovine. In veterinary medicine, a significant fatal coronavirus disease in Felidae is Feline Infectious Peritonitis (FIP). The Internet provided many websites about small animals that present educational content for disease prevention, pets welfare, etc. Upper than 90\% of internet users chooses Google as a search engine. In 2008, Google Company launched Google Insights for search that displaying search trends data. Finally, this company merged Google Insights for Search into Google Trends (GT).We conducted a Google Trends analysis to evaluate search interest in FIP and feline coronavirus in the COVID-19 pandemic. For this purpose, we search two topics, "Feline infectious peritonitis" and "Feline coronavirus" in GT, for collecting data. The duration of study was selected in two periods: January-November 2020, the COVID-19 period and the same period in 2019 for comparison. Comparison of the RSV means between these two periods was performed using two independent samples t-test with assuming unequal variances and two-sided p-value $<0.05$ considered statistically significant. The RSV of "Feline infectious peritonitis" and "Feline coronavirus" topics have significantly increased during the COVID-19 period comparing with the same period in 2019 (pvalue $<0.05$ ). After the COVID-19 pandemic onset, all aspects of our lives were affected by pandemic consequent problems. This global attention to COVID-19 has made a valuable opportunity for presenting the importance of corona viral diseases in any species. The increase of interest in searching about Feline infectious peritonitis and Feline coronavirus indicated that continued global concerns were raised about this infection in felines. The results of this study can be useful for animal health policymakers, veterinarians and journalists to help reduce concerns about FIP and Feline coronavirus by providing educational content on the Internet.
\end{abstract}

Keywords: Coronavirus, COVID-19, Outbreak, Google Trends

\section{Dear Editor}

In December 2019, a respiratory syndrome with an unknown cause was reported in Wuhan, China. The new coronavirus isolated from patients on 7 January 2020 and named Severe Acute Respiratory Syndrome Coronavirus 2 (SARS-CoV-2) (Phelan et al., 2020). The first Coronavirus Disease19 (COVID-19) fatality in China was declared on January 11, 2020. Rapidly after nine days, various countries reported positive cases and consequent problems were started (Majumder and Mandl, 2020). The
World Health Organization declared COVID-19 as pandemic on 11 March 2020; currently, there have been 59,7 million verified cases and 1,4 million deaths worldwide (WHO, 2020).

The COVID-19 pandemic has revealed the importance of the Coronavirus and its diseases to humans around the world (Mertens et al., 2020). Before this global concern, Coronaviridae claimed many lives from animals, especially cats (Paltrinieri et al., 2020; Pedersen, 2009). However, this increase in awareness may help control coronavirus diseases in animals. 
Coronaviruses infected different species of animals, such as feline, canine, swine and bovine (Abdel-Moneim and Abdelwhab, 2020; Decaro and Lorusso, 2020). In veterinary medicine, a significant fatal coronavirus disease in Felidae is Feline Infectious Peritonitis (FIP) (Pedersen, 2009). Feline Infectious Peritonitis Virus (FIPV), the FIP agent, affected several organs, such as the lungs, spleen, liver, kidneys, serosae, central nervous system and eyes. Two main clinical presentations have been seen in FIP, the wet and the dry form. The wet form, protein-rich fibrinous fluids accumulation in body cavities causes' hypovolemia and organ compression (e.g., dyspnea, peristalsis problems, etc.). However, in the dry form, FIPV causes granulomatous lesions in affected organs (Paltrinieri et al., 2020; Pedersen, 2014).

The Internet provided many websites about small animals that present educational content for disease prevention, pets welfare, etc. (Wahl, 2017). User activities on the internet, especially in health, became attractive topics for researchers (Eysenbach, 2011; Infodemiology, 2011; Jahanbin and Rahmanian, 2020; Jahanbin et al., 2021). One of the important subjects for researchers is the level of interest in searching for something among users (McDaid and Park, 2010; Schootman et al., 2015).

Upper than $90 \%$ of internet users chooses Google as a search engine (StatCounter.com, 2019). In 2008, Google Company launched Google Insights for search that displaying search trends data. Finally, this company merged Google Insights for Search into Google Trends (GT) (Matias, 2012). GT is a website that analyzes and visualizes search queries' relative interest in different regions and languages. In this tool, search popularity has been shown by the Relative Search Volume (RSV) index, which has a 0 (absence of interest) to 100 (highest interest) range (Mavragani et al., 2018). The advantages of Google trend analysis in medical research are predicted and surveillance of high prevalent diseases and epidemics. It has been used extensively to the identified outbreaks of infectious diseases (Jahanbin and Rahmanian, 2020). Google Flu Trends collects google search data from 2008 to 2015 in more than 25 countries to forecast flu epidemics (Yang et al., 2015). The implication was that large numbers of Google search queries could be examined to reveal if there was a flu-like illness exists in an area.

We conducted a Google Trends analysis to evaluate search interest in FIP and feline coronavirus in the
COVID-19 pandemic. For this purpose, we search two topics, "Feline infectious peritonitis" and "Feline coronavirus" in GT, for collecting data. The benefit of topic search in GT is including RSV of universal queries related to the topic without limitation in a specific language (Google, 2015). The duration of study was selected in two periods: January-December 2020, the COVID-19 period and the same period in 2019 for comparison. Comparison of the RSV means between these two periods was performed using two independent samples t-test with assuming unequal variances and twosided p-value $<0.05$ considered statistically significant. We used trial version of StatDirect statistical software (http://statdirects.com) for analysis.

Comparisons of the means of RSV and percentage change for related search topics are detailed in Table 1. The RSV of "Feline infectious peritonitis" and "Feline coronavirus" topics have significantly increased during the COVID-19 period (comparing with the same period in 2019 (p-value <0.05). The mean of monthly RSV of two topics during the two investigated period has been presented in Fig. 1. Our investigation in GT is limited by lack of demographics information and data from other search engines.

After the COVID-19 pandemic onset, all aspects of our lives were affected by pandemic consequent problems. This global attention to COVID-19 has made a valuable opportunity for presenting the importance of corona viral diseases in any species. The increase of interest in searching about Feline infectious peritonitis and Feline coronavirus indicated that continued global concerns were raised about this infection in felines. The results of this study can be useful for animal health policymakers, veterinarians and journalists to help reduce concerns about FIP and Feline coronavirus by providing educational content on the Internet. This study is unique in veterinary medicine. However, in medicine and public health, many studies have been conducted to evaluate the interests of searching in the field of health or disease during the COVID-19 pandemic; for example, surveying the google search interest for Facial Plastic Surgery, smoking cessation and Kawasaki disease during the COVID-19 pandemic period (Dey and Zhao, 2020; Duggan et al., 2020; Heerfordt and Heerfordt, 2020).

The limitation of this investigation are that search interest data are limited to Google users and google trend does not report search results with an exact number but reports relatively.

Table 1: Relative search volume during the COVID-19 period vs previous year

\begin{tabular}{lllllll}
\hline Search topic & RSV, 2020, mean (SD) & RSV, 2019, mean (SD) & Percent change & Difference in means & $95 \%$ CI & p-value \\
\hline Feline infectious peritonitis & $64.76(4.65)$ & $52.47(14.58)$ & 23.40 & 12.28 & {$[7.88,16.68]$} & $<0.001^{\mathrm{b}}$ \\
Feline coronavirus & $19.69(23.11)$ & $1.84(0.55)$ & 965.88 & 17.84 & {$[10.96,24.72]$} & $<0.001^{\mathrm{b}}$ \\
\hline
\end{tabular}

${ }^{\mathrm{b}}$ Two-sided p-value $<0.05$, statistically significant. RSV, Relative Search Volume. SD, Standard Deviation. CI, Confidence Interval 


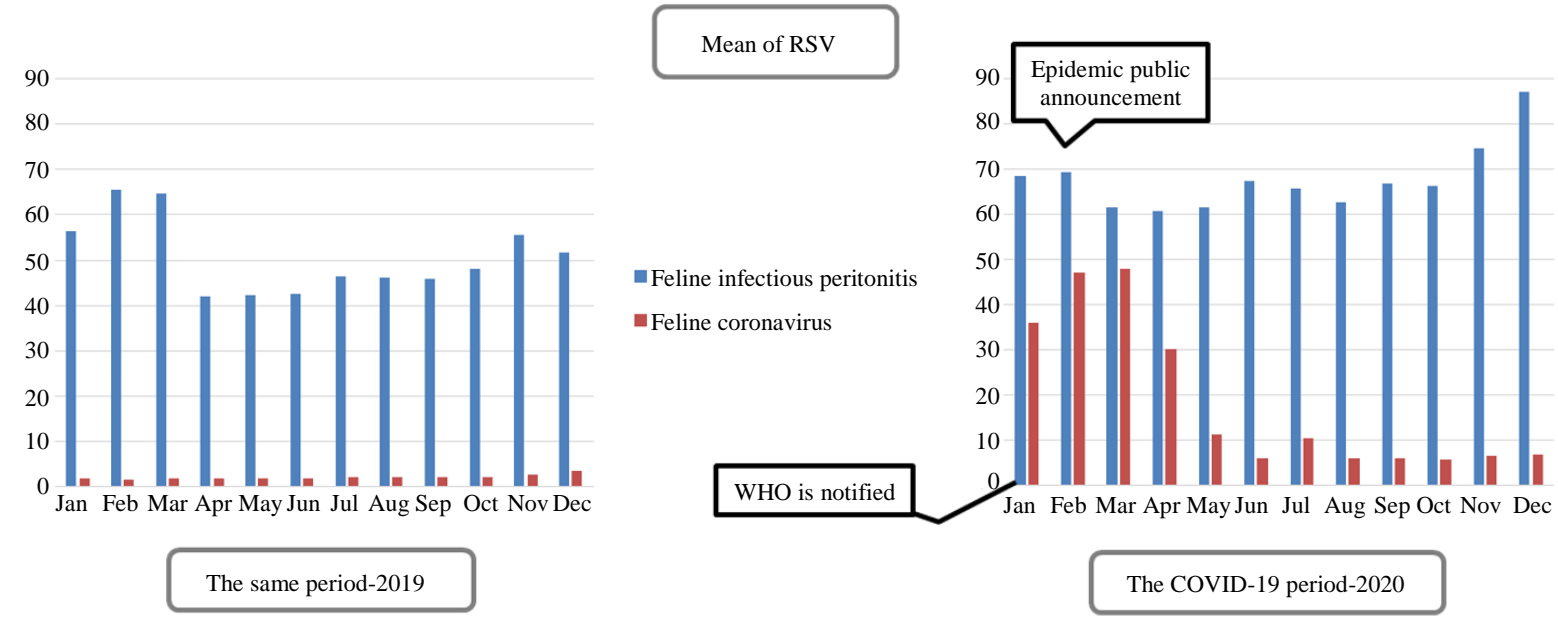

Fig. 1: (a) Popularity in Google searches for "Feline infectious peritonitis" and "Feline coronavirus", January-December 2020 (the COVID-19 period) and the same period in 2019. RSV scores ranged from 0 (lack of interest) to 100 (highest interest) and presented monthly.

\section{Acknowledgement}

The authors hereby would like to express their gratitude and appreciation to Dr. Mohammad Hasan Rabiee for collaboration and providing advice on the implementation in this study.

\section{Authors' Contributions}

Mohammad Jokar: Organized the study, proposal of study writing, data gathering, manuscript preparation, data analysis and interpretation of data, literature search and final revision of the study.

Vahid Rahmanian: Organized the study, proposal of study writing, analysis and interpretation of data, manuscript editing, final revision of the study content and final approval of the revision the manuscript.

Nader Sharifi, Narges Rahmanian and Hekmatollah Khoubfekr: Data gathering, Design of study, literature search and final approval of the version of the manuscript.

\section{Ethics}

The authors of this study followed the ethical principles, including guidance on authorship, avoiding redundant (duplicate) publication, avoiding plagiarism, transparency, ensuring accuracy that potential complications.

\section{References}

Abdel-Moneim, A. S., \& Abdelwhab, E. M. (2020). Evidence for SARS-CoV-2 infection of animal hosts. Pathogens, 9(7), 529. https://doi.org/10.3390/pathogens9070529
Decaro, N., \& Lorusso, A. (2020). Novel human coronavirus (SARS-CoV-2): A lesson from animal coronaviruses. Veterinary Microbiology, 108693. https://doi.org/10.1016/j.vetmic.2020.108693

Dey, M., \& Zhao, S. S. (2020). COVID-19 and Kawasaki disease: an analysis using Google Trends. Clinical Rheumatology, 39(8), 2483-2484. https://doi.org/10.1007/s10067-020-05231-z

Duggan, R. P., Tran, J. P., \& Phillips, L. G. (2020). Interest in Plastic Surgery during COVID-19 Pandemic: A Google Trends Analysis. Plastic and Reconstructive Surgery-Global Open, 8(10), e3268. https://doi.org/10.1097/GOX.0000000000003268

Eysenbach, G. (2011). Infodemiology and infoveillance: Tracking online health information and cyberbehavior for public health. American Journal of Preventive Medicine, 40(5), S154-S158. https://doi.org/10.1016/j.amepre.2011.02.006

Google. (2015). Trends Help. https://support.google.com/trends/answer/4355164? $\mathrm{hl}=\mathrm{en} \& \mathrm{rd}=1$

Heerfordt, C., \& Heerfordt, I. M. (2020). Has there been an increased interest in smoking cessation during the first months of the COVID-19 pandemic? A Google Trends study. Public Health, 183, 6. https://doi.org/10.1016/j.puhe.2020.04.012

Infodemiology, E. G. (2011). Infoveillance. American Journal Prevention Medicine, 40(5), S154.

Jahanbin, K., \& Rahmanian, V. (2020). Using Twitter and web news mining to predict COVID-19 outbreak. Asian Pacific Journal of Tropical Medicine, 13(8), 378.

https://doi.org/10.4103/1995-7645.279651 
Jahanbin, K., Rahmanian, V., \& Rahmanian, F. (2020). Predicting COVID-19 in Iran using automate web crawling. Pakistan Journal of Medical \& Health Sciences, 14(4), 987-91.

Majumder, M., \& Mandl, K. D. (2020). Early transmissibility assessment of a novel coronavirus in Wuhan, China. China (January 23, 2020). https://doi.org/10.2139/ssrn.3524675

Matias, Y. (2012). Insights into what the world is searching for-the new Google Trends. Available at insidesearch. Blogspot. ca/2012/09/insights-intowhat-world-is-searching.html.

http://insidesearch.blogspot.co.il/2012/09/insightsinto-what-world-is-searching.html

Mavragani, A., Ochoa, G., \& Tsagarakis, K. P. (2018). Assessing the methods, tools and statistical approaches in Google Trends research: systematic review. Journal of Medical Internet Research, 20(11), e9366. https://doi.org/10.2196/jmir.9366

McDaid, D., \& Park, A. L. (2010). Online health: untangling the web. http://eprints.lse.ac.uk/32690/

Mertens, G., Gerritsen, L., Duijndam, S., Salemink, E., \& Engelhard, I. M. (2020). Fear of the coronavirus (COVID-19): Predictors in an online study conducted in March 2020. Journal of Anxiety Disorders, 74, 102258. https://doi.org/10.1016/j.janxdis.2020.102258

Paltrinieri, S., Giordano, A., Stranieri, A., \& Lauzi, S. (2020). Feline infectious peritonitis (FIP) and coronavirus disease 19 (COVID-19): Are they similar?. Transboundary and Emerging Diseases. https://doi.org/10.1111/tbed.13856
Pedersen, N. C. (2009). A review of feline infectious peritonitis virus infection: 1963-2008. Journal of Feline Medicine and Surgery, 11(4), 225-258. https://doi.org/10.1016/j.jfms.2008.09.008

Pedersen, N. C. (2014). An update on feline infectious peritonitis: virology and immunopathogenesis. The Veterinary Journal, 201(2), 123-132. https://doi.org/10.1016/j.tvj1.2014.04.017

Phelan, A. L., Katz, R., \& Gostin, L. O. (2020). The novel coronavirus originating in Wuhan, China: challenges for global health governance. JAMA, 323(8), 709-710. https://doi.org/10.1001/jama.2020.1097

Schootman, M., Toor, A., Cavazos-Rehg, P., Jeffe, D. B., McQueen, A., Eberth, J., \& Davidson, N. O. (2015). The utility of Google Trends data to examine interest in cancer screening. BMJ Open, 5(6), e006678. https://doi.org/10.1136/bmjopen-2014-006678

StatCounter.com. (2019). StatCounter Global StatsBrowser, OS, Search Engine including Mobile Usage Share.https://gs.statcounter.com/

Wahl, I. Y. (2017). Implying pet owners' ideas into veterinary practices' websites (Master's thesis). https://dspace.library.uu.nl/handle/1874/369600

WHO. (2020). Coronavirus Disease (COVID-19). https://www.who.int/emergencies/diseases/novelcoronavirus-2019

Yang, S., Santillana, M., \& Kou, S. C. (2015). Accurate estimation of influenza epidemics using Google search data via ARGO. Proceedings of the National Academy of Sciences, 112(47), 14473-14478. https://doi.org/10.1073/pnas.1515373112 